\title{
Naltrexone plus cognitive behavioural therapy led to fewer drinking relapses in outpatients with alcoholism
}

Anton RF, Moak DH, Waid LR, et al. Naltrexone and cognitive behavioral therapy for the treatment of outpatient alcoholics: results of a placebo-controlled trial. Am J Psychiatry 1999 Nov;156:1758-64.

QUESTION: In outpatients with alcoholism who are receiving cognitive behavioural therapy (CBT), is naltrexone more effective than placebo?

\section{Design}

Randomised \{allocation concealed $*\} \uparrow$, blinded (patients and outcome assessors)*, placebo controlled trial with 12 weeks of follow up.

\section{Setting}

Alcohol Research Center, Medical University of South Carolina, South Carolina, USA.

\section{Patients}

131 patients between 21 and 65 years of age (mean age $43 \mathrm{y}, 71 \%$ men) referred or replying to a newspaper advertisement for outpatient treatment of DSM-III-R confirmed alcohol dependence. Other inclusion criteria were consumption of $\geq 5$ drinks/day in the previous 30 days and loss of control of drinking, and ability to maintain sobriety for $\geq 5$ days before study entry. Exclusion criteria were previous inpatient detoxification in which medication was taken for alcohol withdrawal, other drug abuse or dependence, a major psychiatric disorder, a serious or unstable medical condition, use of psychotropic or antiseizure medications or disulfiram, or liver function test results $>2.5$ times normal. 132 patients were randomised but 1 patient did not return.

\section{Intervention}

68 patients were allocated to $50 \mathrm{mg} /$ day of naltrexone and 63 were allocated to placebo. All patients were required to attend 12 weekly sessions of individual manual guided CBT.

\section{Main outcome measures}

Drinking relapse ( $\geqslant 5$ drinks/day for men and $\geqslant 4$ for women), time to relapse, number of drinks per drinking day, craving, and adverse events.

\section{Main results}

Analysis was by intention to treat. End point data were available for $98.5 \%$ of patients. At the end of the study, fewer patients in the naltrexone group had relapsed compared with those in the placebo group $(\mathrm{p}=0.01)$ (table). Time to relapse was greater in patients in the naltrexone group and mean number of drinks per drinking day were fewer (table). Patients in the naltrexone group had a lower score on the resistance/control impairment factor of the Obsessive Compulsive Drinking Scale than those in the placebo group $(p=0.03) .1$ patient from each of the study groups dropped out because of a stated adverse effect.

\section{Conclusion}

In outpatients with alcoholism, naltrexone in conjunction with cognitive behavioural therapy led to fewer drinking relapses than did placebo.

*See glossary.

$\dagger$ Information provided by the author.

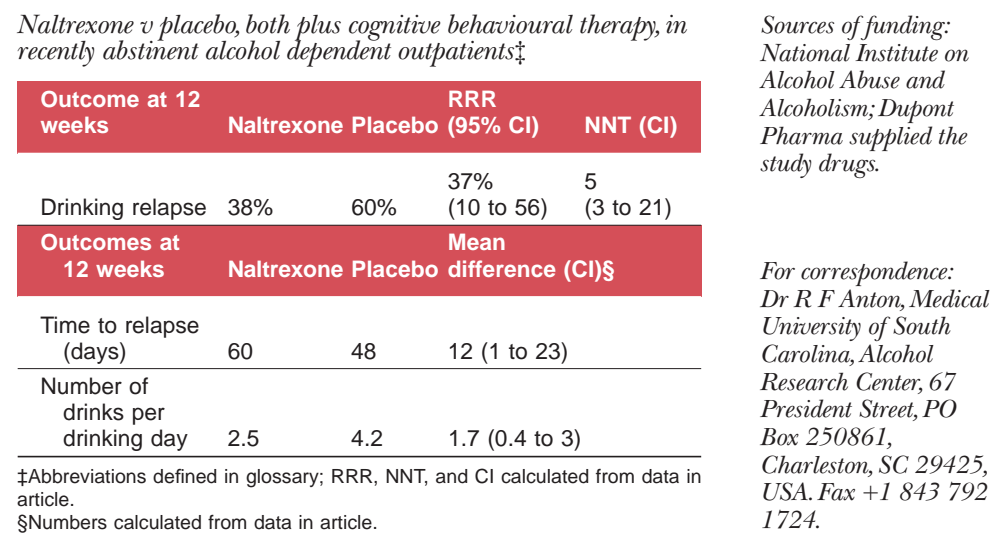

\section{COMMENTARY}

The earliest published study on the use of the opioid antagonist, naltrexone, in preventing relapse in alcohol dependence was conducted in severely affected patients. ${ }^{1}$ Subsequent studies have been in slightly less severe samples. The patient population in this study by Anton $e t$ al was specifically defined as a less severe group in that those who had had a previous medicated inpatient detoxification were excluded. In addition, a proportion (unspecified) of the patients were recruited via newspaper advertisements, a method which may possibly select less medically severe subjects than typical clinic referrals. The patients were also a socially stable group: $68 \%$ married and $81 \%$ in full time employment, reflected in the exceptionally high follow up rate $(98.5 \%)$.

The treatment effects summarised in the above abstract are impressive. One caveat should be made. More patients reported nausea on naltrexone (34\%) than on placebo $(14 \%)$. There was no test to determine whether the blindness of patients or research assistants who saw the patients weekly was intact at the end of the study. Also, it is not reported whether an objective test such as a breathalyser was part of the follow up assessments, but data from serum carbohydrate deficient transferrin and serum gamma glutamyl transferase did not show an advantage to the naltrexone group.

It is emerging that, in conjunction with coping skills treatment or CBT, opiate antagonists improve outcome measured as relapse to heavy drinking more effectively than outcome measured as complete abstinence. ${ }^{23}$ In this study, complete abstinence for the 12 weeks was attained by $47 \%$ of the naltrexone group and $33 \%$ of the placebo group-but this difference was not statistically significant. Instead, a statistical difference was seen in reducing relapse to heavy drinking and total alcohol consumed per drinking day. This study also found that the mean time between a first relapse and a second relapse was doubled (14 days for naltrexone patients instead of 6 days). The CBT offered in this study would not have demanded abstinence as an exclusive goal. Thus, Anton et al conclude that naltrexone reinforces the strategy in CBT of preventing a slip turning into a fullblown relapse.

Some questions remaining include: (1) does naltrexone reduce relapse for patients receiving only brief psychological treatment? And (2) is at least a brief period of abstinence necessary before starting an opiate antagonist?

Jonathan Chick, MPhil, FRCPE, FRCPsych Royal Edinburgh Hospital Edinburgh, UK

1 Volpicelli JR, Alterman AI, Hayashida M, et al. Naltrexone in the treatment of alcohol dependence. Arch Gen Psychiatry 1992;49:876-80.

2 O'Malley SS, Jaffe AJ, Chang G, et al. Naltrexone and coping skills therapy for alcohol dependence. Arch Gen Psychiatry 1992;49:881-7.

3 Mason BJ, Salvato FR, Williams LD, et al. A double-blind placebo-controlled study of oral nalmefene for alcohol dependence. Arch Gen Psychiatry 1999;56:719-24. 